\title{
A 2-year study of the efficacy and tolerability of prenylamine in the treatment of angina pectoris
}

\author{
NeIL CARDOE \\ M.B., B.S., M.R.C.P. \\ Norfolk and Norwich and Cromer and District Hospitals
}

\begin{abstract}
Summary
Twelve out-patients with established angina pectoris who had previously participated in a double-blind trial of prenylamine lactate continued to record their daily anginal attack rate and glyceryl trinitrate consumption over the subsequent 18 months. The improvement noted while taking prenylamine during the trial was maintained and a further double-blind study confirmed that this was, in fact, due to the administration of the drug, which was again shown to be significantly more effective than identical placebo in the prophylaxis of angina pectoris.
\end{abstract}

\section{Introduction}

Prenylamine lactate (N-(3,3-diphenylpropyl) methyl phenethylamine) has been shown to reduce the catecholamine content of sympathetically innervated organs. This is due to inhibition of uptake of noradrenaline by the storage granules (Schöne \& Lindner, 1960; Juorio \& Vogt, 1963; Carlsson \& Waldeck, 1968; Euler, Stjarne \& Lashajko, 1964; Euler \& Stjarne, 1968). No beta-blocking action has been demonstrated (Hodge, 1969; Fleckenstein, 1968; Lindner, 1964). Studies of the effects of prenylamine lactate in man have demonstrated a slowing of the resting heart rate and reduction in post-exercise tachycardia (Kirchhoff \& Herter, 1968; Pippig, Schneider \& Hochrein, 1962; Abrahamsen, 1963; Batson, 1970, personal communication). Doubleblind studies have shown prenylamine lactate to be effective in reducing the incidence of anginal attacks (Donat \& Schlosser, 1966; Kappert, 1965; Luisada \& Neumann, 1963; Stoker, 1968; Cloarec, 1968). In a previous double-blind study (Cardoe, 1968) the efficacy of prenylamine lactate in reducing the anginal attack-rate was confirmed. This paper reports the long-term follow-up of patients taking part in that study and a further double-blind trial comparing prenylamine lactate with identical placebo in the same group of patients.

\section{Material and methods}

\section{(a) 18 month treatment period}

Twelve of the thirteen patients in the previous double-blind trial continued to record their daily anginal attack rate and glyceryl trinitrite (GTN) consumption and continued to take prenylamine lactate in the same dose. However, after a short time it was possible to reduce the dose by $60 \mathrm{mg} /$ day in five cases, as the original dose proved unnecessary for satisfactory control. Thus, the dose range of prenylamine lactate was as follows: $120 \mathrm{mg} /$ day in three cases, $180 \mathrm{mg} /$ day in six cases and $240 \mathrm{mg} /$ day in the remaining three cases. No other anti-anginal agent other than GTN $0.5 \mathrm{mg}$ BP was given at any time. As in the previous study, to be recorded an anginal attack had to be severe enough to interfere with activity at the time of the attack.

\section{(b) Double-blind trial}

After treatment for approximately 18 months, a further double-blind study was instituted. The trial consisted of four treatment periods, each of 4 weeks duration, two periods of prenylamine lactate administration in the same dosage as before and two periods in which the patients received identical placebo. The order of administration was randomized in such a way that no two consecutive periods were similar. Neither the physician nor the patients knew the identity of the tablets given in any period and in order to avoid the possible effect of 'suggestion' the patients were unaware that a placebo was being introduced. The patients were asked to continue recording the anginal attack rate and GTN consumption and were reviewed at 4-weekly intervals. At each visit the anginal attack rate and GTN consumption was noted, the latter being checked by counting the number of tablets utilized since the previous visit. Resting pulse rate and blood pressure were also recorded and any side effects noted. In addition, at the last visit haemoglobin, total and differential white cell count, blood urea, SGOT and SGPT values were determined.

Of the patients in the study, all had typical anginal pain and all but one had electrocardiographic signs of myocardial ischaemia.

\section{Results}

\section{(a) 18 month treatment period}

The mean fortnightly anginal attack-rate over the 


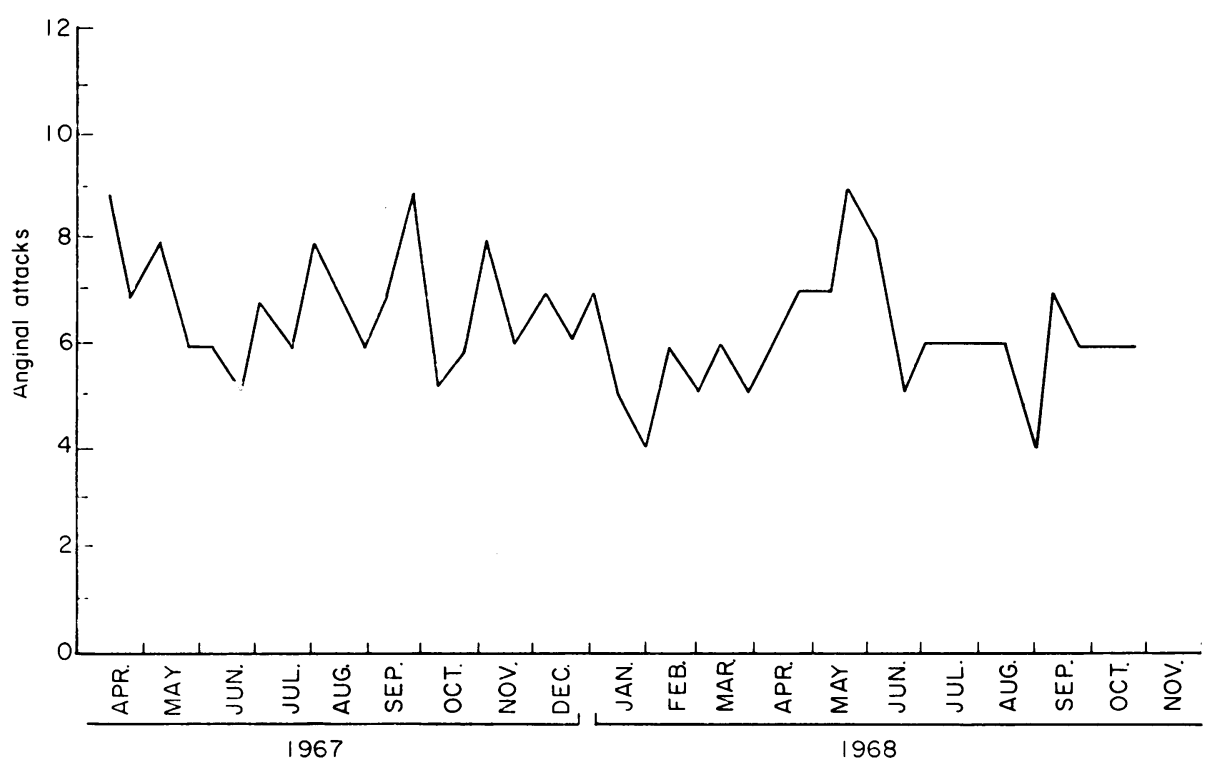

Fig. 1. Mean fortnightly anginal attack rate during 18 month treatment with prenylamine.

period April 1967 to October 1968 is shown in Fig. 1. The figures for GTN consumption were identical.

No side effects attributable to prenylamine occurred.

\section{(b) Second double-blind study}

In order to confirm that the sustained improvement in this group of patients was, in fact, due to the administration of prenylamine, a second doubleblind study was instituted. The results of this study are shown in Table 1. This shows that more attacks of angina occurred when the patients were taking placebo tablets than when they were on the active preparation. The column headed ' $\mathrm{P}-\mathrm{A}$ ' gives the difference in attack rate. The results on active and placebo tablets for all patients were examined statistically by Student's paired $t$-test. This gave a mean difference (placebo minus prenylamine) of 12.8 and ' $t$ ' $=3.95$ with eleven degrees of freedom. This value is significant at the 0.01 level of probability.

The consumption of GTN tablets closely parallels the anginal attack-rate and the difference between active and placebo periods is also significant at the 0.01 level of probability. The resting pulse rate and blood pressure readings shown are those taken at the end of the second period of administration of prenylamine and at the end of the second placebo period. No readings were available for patient No. 1 while on the active preparation and this case has, therefore, been omitted from the calculations. It can be seen that the pulse rate tends to be lower during treatment with prenylamine but the difference is not statistically significant. Similarly, there is no signi- ficant difference in blood pressure values between active and placebo periods.

All patients continued to tolerate the drug well and no side-effects occurred. On the occasion of their last visit to the clinic a blood sample was taken for haemoglobin, total and differential white cell count, blood urea, SGOT and SGPT. The results of these estimations were within normal limits in all cases and further, showed no significant difference from those obtained during the first double-blind study 2 years previously.

\section{Discussion}

A review of the literature reveals little evidence of the long-term efficacy of anti-anginal preparations. This study demonstrates that the improvement in anginal attack-rate produced by prenylamine during the first double-blind trial was maintained over a period of 18 months. The mean fortnightly anginal attack-rate of this group of patients over the full period of study is shown in Fig. 2. It can be seen that during the preliminary observation period before the first double-blind trial this value was 27.6. During the placebo periods of the study this fell to $17 \cdot 3$. With the administration of prenylamine a further fall to 8.8 occurred and was subsequently maintained at a mean level of 6.4 for the following 18 months. During the second double-blind trial a rise to 10.3 occurred when the patients received placebo tablets but this was reduced to 7.0 on the administration of prenylamine.

The differences in anginal attack rate between the placebo periods and active periods in both double- 


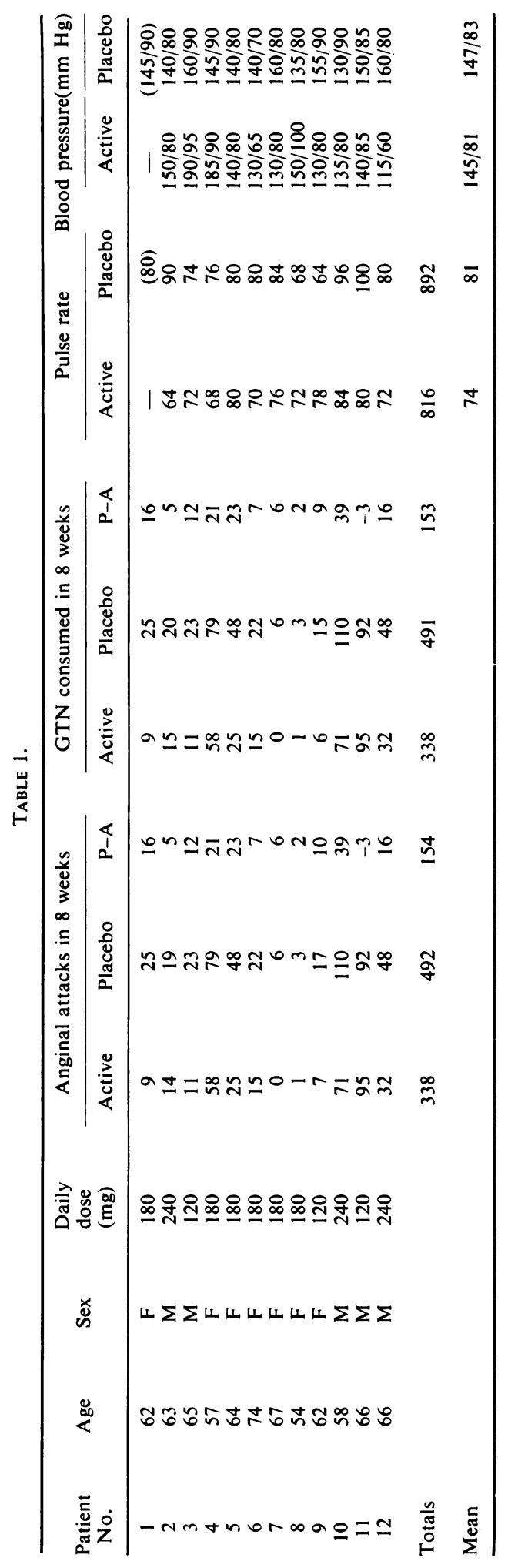




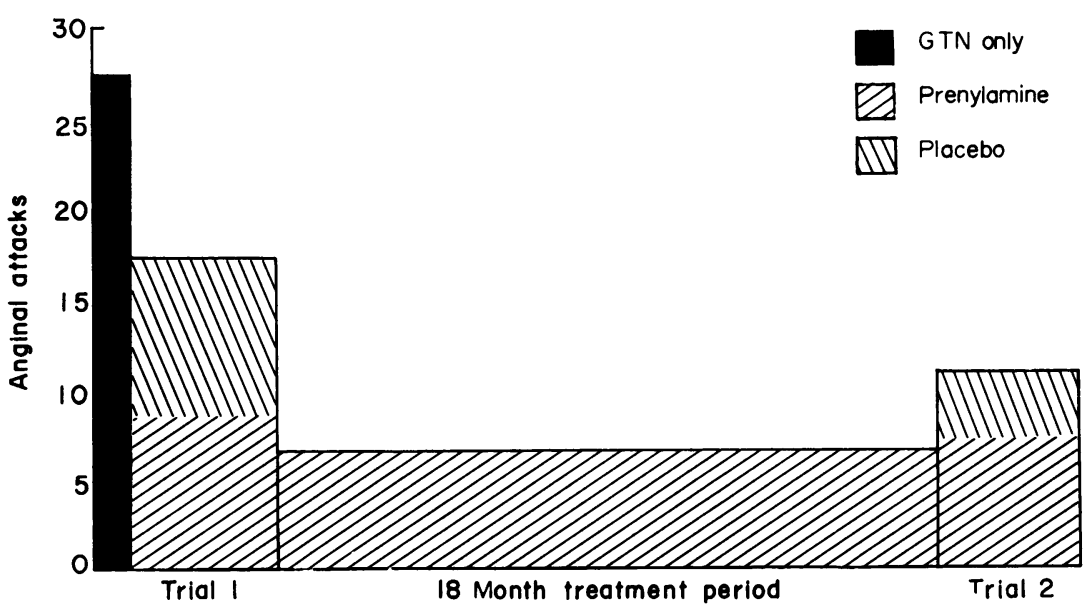

Fig. 2. Mean fortnightly anginal attack rate over a period of 24 months.

blind studies are, of course, statistically significant, as is the difference in mean fortnightly attack-rate between the 18-month treatment-period and the placebo periods of the second double-blind study. Therefore, it is clear that the improvement achieved with prenylamine in the first double-blind study and maintained over the following 18 months was, in fact, due to the administration of the drug.

It is of interest to note that in the 18-month treatment-period the variation in incidence of attacks is relatively small and is unrelated to seasonal variation in temperature (see Fig. 1). This is in contradistinction to the findings of Aubert et al. (1970), who found a close correlation between anginal attack rate and outdoor temperature, and may be due to the patients realising that exposure to cold could precipitate an attack and thus avoiding such exposure whenever possible.

Further, it may be seen that the passage of time had no significant effect on the attack-rate, the average fortnightly attack-rate in the first 9 months being 6.8 and in the second 9-month period 6.0.

As noted by Wilson et al. (1969), in their trial of oxprenolol, it became obvious during the course of this study that the customary quantitative results did not offer a true reflection of the efficacy of the active preparation. This was largely due to the fact that patients receiving the drug experienced improvement in effort tolerance and thus undertook a greater degree of exercise, frequently to the point of precipitating an anginal attack. Such an improvement is not apparent in the data, which only reflect quantitative results. This is exemplified by two patients in this study who, because of their improved effort tolerance on prenylamine, undertook relatively strenuous holidays. Their anginal attack-rate during this time differed little from before but obviously their capacity for exercise was greatly improved.
Finally, it is of interest to comment on the safety and tolerability of prenylamine. In this group of patients who received the drug over a total period of 2 years, no side-effects attributable to its administration occurred, nor was there any undesirable effect on haemoglobin, white cell count, blood urea, SGOT or SGPT. No serious complications, i.e. cardiac failure or bronchospasm, which have been reported in association with the use of adrenergic beta-blocking agents (Stephen, 1966; Besterman, 1966; Macdonald, Ingram \& McNeil, 1967) were noted. This is probably due to the different mechanism whereby prenylamine achieves a reduction in sympathetic drive to the myocardium.

\section{Acknowledgments}

My thanks are due to Dr M. E. Kennedy, Medical Department, Hoechst Pharmaceuticals and his colleagues for supplies of prenylamine lactate (Synadrin 60) and to the nursing and secretarial staff of the Cromer and District Hospital.

\section{References}

Abrahamsen, A.M. (1963) Cardiac catheterization studies following injection of Synadrin. Acta Medica Scandinavica, 174(3), 265.

Aubert, A., Nyberg, G., Slaastad, R. \& Tueldflaat, L. (1970) Prophylactic treatment of angina pectoris. British Medical Journal, 1, 203.

Besterman, E.M.M. (1966) Precipitation of asthma in chronic bronchitis. American Journal of Cardiology, 18, 475.

Cardoe, N. (1968) Prenylamine lactate (Synadrin) in patients with angina pectoris. British Journal of Clinical Practice, 22(7), 299.

Carlsson, A. \& Waldeck, B. (1968) On the mechanism of action of prenylamine on tissue monoamines. Biochimica Applicata, 14 (Suppl. 1), 41.

Cloarec, M. (1968) Prenylamine. Its use in Coronary Disease. La Prénylamine en Thérapeutique. Colloque de Mcnaco, March 1-2, 1968, pp. 53-65. S.P.E.I., Département Editions Médicales, Paris. 
Donat, K. \& Schlosser, G.A. (1966) Problems in the treatment of angina pectoris. Medizinische Klinik, 61(9), 352.

EUler, U.S.v. \& Lishajko, F. (1968) Observations on the actions of prenylamine (Segontin) in vivo and on adrenergic transmitter granules. Biochimica Applicata, 14 (Suppl. 1), 17.

Euler, U.S.v., STJARNe, L. \& Lishajko, F. (1964) Effects of reserpine, segontin and phenoxybenzamine on the catecholamines and ATP of isolated nerve and adrenomedullary storage granules. Life Sciences, 3(1), 35.

Fleckenstein, A., Döring, H.J. \& KAMmermeier, $H$. (1968) Influence of prenylamine on the utilisation of high energy phosphates in cardiac muscle. Biochimica Applicata, 14 (Suppl. 1), 323.

HODGE, R.L. (1969) Investigation of a possible beta-blocking action of prenylamine in man. Australian Journal of Experimental Biology and Medicine Science, 47, 651.

JuORIO, A.V. \& VoGT, M. (1963) The effect of prenylamine on the metabolism of catecholamines and 5-hydroxytryptamine in brain and adrenal medulla. British Journal of Pharmacology, 24, 566.

KAPPERT, A. (1965) Double-blind trial with high doses of segontin in angina pectoris. Zeitschrift Therapie, 3(2), 82.

KIRCHHOFF, H.W. \& HERTER, B.L. (1968) Investigations on the effect of segontin on various circulatory parameters before and during exercise. International Journal of Clinical Pharmacology, 1(4), 302.
LINDNER, E. (1964) The effect of beta-sympatholytic agents , on cardiac dynamics. Verhandlungen der Deutschen Gesellschaft für Kreislauf-forschung, 70 Kongress, pp. 202-205, 1964.

Luisada, A.A. \& Neumann, M. (1963) Double-blind study with 'coronary drugs' in old patients. Acta Sec. Conv. Med. Int. Hung. pp. 94-98.

MaCdonald, A.G., IngRam, C.G., McNeIL, R.S. (1967) The effect of propranolol on airway resistance. British Journal of Anaesthesia, 39, 919.

PipPig, L., Schneider, K.W. \& Hochrein, H. (1962) Studies on the behaviour of the heart minute volume and other hemodynamic values under the effect of $\mathrm{N}-3^{1}$ Phenyl - propyl - (2) - 1, 1 - diphenylpropyl - (3) - amine. Medizinische Klinische, 57, 1475.

SChÖNE, H.-H. \& LindNer, E. (1960) The effects of N-((31Phenylpropyl - (21)) - 1,1 - diphenylpropyl - (3) - amine on serotonin and norepinephrine metabolism: ArzneimittelForschung, 10, 583.

StePHEN, S.A. (1966) Unwanted effects of proprano.ol. American Journal of Cardiology, 18, 463.

STOKER, J.B. (1968) Effect of prenylamine in angina pectoris. British Journal of Clinical Practice, 22, 384.

Wilson, D.F., Watson, O.F., Peel, J.S. \& Turner, A.S. (1969) Trasicor in angina pectoris: A double-blind trial. British Medical Journal, 2, 155. 